\title{
BMJ Open Relationship between receipt of substitutable for-fee vaccines and completion of the expanded programme on immunisation: a cross-sectional study in Fujian, China
}

\author{
Jiang-Nan Wu, ${ }^{1}$ Da-Jin Li, ${ }^{1}$ Yong Zhou, ${ }^{2}$ Mei-Rong Du, ${ }^{1}$ Hai-Lan Piao ${ }^{1}$
}

To cite: Wu J-N, Li D-J, Zhou Y, et al. Relationship between receipt of substitutable for-fee vaccines and completion of the expanded programme on immunisation: a cross-sectional study in Fujian, China. BMJ Open 2017;7:e015666. doi:10.1136/ bmjopen-2016-015666

- Prepublication history for this paper is available online. To view, these files please visit the journal online (http://dx.doi org/10.1136/bmjopen-2016015666).

Received 23 December 2016 Revised 11 May 2017 Accepted 12 May 2017
CrossMark

\section{${ }^{1}$ Department of Clinical} Epidemiology, Obstetrics and Gynecology Hospital of Fudan University, Shanghai, China ${ }^{2}$ Department of Expanded Programme on Immunization, Fujian Provincial Center for Disease Control and Prevention, Fuzhou, China

Correspondence to Dr Jiang-Nan Wu; wjnhmm@ 126.com

\section{ABSTRACT}

Objective The aim of this study was to evaluate the relationship between receipt of the substitutable-forfee vaccines (SFV) and completion of the expanded programme on immunisation (EPI).

Design and settings A cross-sectional study was conducted in Fujian province, China.

Participants Children who were born from 1 September 2009 to 31 August 2011, and who had been residing in the township for at least 3 months, were randomly recruited from 34 townships.

Main outcomes measures Outcomes were completion rate of the EPI and coverage rate of the SFV.

Results The study included 1428 children, of whom 1350 (94.5\%) finished the EPI and $282(19.7 \%)$ received at least one dose of the SFV. Administration of the SFV was associated with an increased likelihood of completing the EPI $(\mathrm{OR}=3.2,95 \% \mathrm{Cl} 1.3$ to 7.6 in the total sample and $\mathrm{OR}=4.0,95 \% \mathrm{Cl} 1.7$ to 9.6 in the subsample of children in regions with the SFV accessibility). The impact of the SFV administration on completion of the EPI was larger among children whose parents have junior school education or less $(97.8 \%$ and $97.9 \%$ vs $92.5 \%$ and $91.9 \%$, both $\mathrm{p}<0.001)$ and among those with a timely hepatitis $\mathrm{B}$ vaccine first dose $(98.5 \%$ vs $94.0 \%, p<0.001)$.

Conclusions Receipt of SFV is associated with increased likelihood of completion of the EPI in Fujian, China.

\section{INTRODUCTION}

China has an expanded programme on immunisation (EPI) which includes one dose of Bacille Calmette-Guerin vaccine (BCG), three doses of hepatitis $B$ vaccine (HepB), four doses of oral live attenuated polio vaccine (OPV), four doses of acellular pertussis diphtheria tetanus vaccine (DTaP), two doses of measles-containing vaccine (MCV), two doses of group A meningococcal polysaccharide vaccine (MPSV-A), two doses of live-attenuated Japanese encephalitis vaccine (JE-L), one dose of live-attenuated hepatitis A vaccine (HepA-L), one dose of diphtheria
Strengths and limitations of this study

- This is the first study exploring the association between substitutable for-fee vaccines (SFV) administration and the expanded programme on immunisation completion in China.

- The subjects were selected randomly by lot quality assurance sampling from townships which were randomly extracted by the probability proportional to size sampling method, as well as a high response rate $(100 \%)$ in the survey; therefore, the sample had a good representation.

- The findings may help target vulnerable children and improve the implementation of the SFV policy in China.

- The sample size was not large enough, and we were not able to control all potential confounding factors in the analyses, both may limit the interpretation of some results.

tetanus vaccine (DT) and two doses of meningococcal $\mathrm{A}+\mathrm{C}$ polysaccharide vaccine (MPSV-AC). ${ }^{1}$ The EPI vaccines are mostly produced by state-owned pharmaceutical companies and are provided to the public as free vaccines. In addition, parents can choose to purchase variants that are for-fee vaccines, which are mostly manufactured by private enterprises based in China or are imported from abroad. The for-fee vaccines can be further divided into two types, normal and substitutable for-fee vaccines $(\mathrm{SFV}) .^{2}{ }^{3}$ For example, pneumococcal conjugate vaccine is a normal for-fee vaccine, while inactivated polio vaccine (IPV) is an SFV and can be a substitute for OPV.

Whether to carry out service of the SFV for children has been controversial in China. On the one hand, given worries that the SFV service may cause social injustice and affect the completion of the EPI, the National Health and Family Planning Commission of 
the People's Republic of China (China NHFPC) opposed the SFV service. ${ }^{4}$ In some areas, the Health Bureau even prohibited staff in immunisation clinics (IC) from administering the SFV vaccination to children. On the other hand, parents always think the SFV was better than EPI vaccines (eg, safer and more effective), the opinions have been strengthened since the adverse events after the EPI immunisation was reported. ${ }^{56}$ Hence, parents may select the SFV once they can afford the fee, and/or they think it is necessary (eg, children are not in good condition for EPI vaccines). Moreover, vaccine providers may recommend the SFV once they thought the SFV was safer than EPI vaccines, worried about the troubles caused by adverse events following immunisation (AEFI) (eg, vaccine-associated paralytic poliomyelitis after OPV immunisation $)^{7}$ and were under pressure to create income for the unit. ${ }^{8}$ Therefore, the SFV immunisation was widely performed in China. It was estimated that $18.05 \%$ of children had received at least one dose of the SFV nationally, and the coverage of the SFV was higher among children in eastern areas than that in western regions. ${ }^{2}$

Previous studies primarily focused on describing coverage of normal for-fee vaccines (such as pneumococcal conjugate vaccine and influenza vaccine) and their determinants, ${ }^{8-10}$ and investigating coverage of the SFV and/or its substitution rate to the EPI vaccines. ${ }^{211} 12$ However, no studies to date were conducted to probe the relationship between the SFV vaccination and the EPI completion. Knowing the relationship is important for guidance and management of the SFV service, such as, if the SFV were conductive to complete the EPI for children, the China NHFPC may improve the policy of SFV and encourage vaccination with the SFV for children who can afford the vaccine fee.

Furthermore, parents who choose to have their children received the SFV might not only show a greater disposable income (because the SFV requires an out-of-pocket payment $)^{2}$ but also have higher awareness of immunisation (because they chose the SFV instead of the EPI vaccines). Better economic capacities and higher vaccination awareness were significantly related to completion of the EPI vaccine series. ${ }^{1314}$ Moreover, previous studies had revealed that children who had initiated a delayed first dose of $\mathrm{HepB}^{1}$ and whose parents have lower educational levels were less likely to complete the EPI. ${ }^{15}$ Therefore, it is reasonable to hypothesise that(1) children receiving the SFV might promote their completion of the EPI and (2) the relationship between the SFV administration and the EPI completion might be modified by parents' education level and initiation time of the НерB first dose. We aimed at assessing coverage rates of the SFV and verifying the hypotheses.

\section{METHODS}

\section{Participants and design}

The cross-sectional study was part of a national EPI coverage rate investigation and was conducted in
December 2013 in Fujian, China. Details of the national survey have been described elsewhere. ${ }^{16}{ }^{17}$ In brief, Chinese children aged 2-4 years at the time of the survey (born from 1 September 2009 to 31 August 2011) and who had been residing in the township for at least 3 months were included as target subjects and were randomly selected for the survey. First, a township with a population less than 10000 was combined with an adjacent township to ensure adequate sample for survey, resulting in 1004 combined townships with a population of $\geq 10000$ from the 1058 townships in Fujian province. Second, 34 townships were randomly selected by the probability proportional to size sampling method from the 1004 townships. For each township sampled, five villages (the village where the township government was located and another four randomly selected villages) were sampled as village-level survey sites. Finally, at the village-level survey sites, lot quality assurance sampling was used to sample households for survey.

Parents or guardians of the children were interviewed by a standardised questionnaire which was set by China Center for Disease Control (CDC). The questionnaire contained two major items, including basic information and vaccination history. Measures of family socioeconomic situations (home address, types of living areas and education level of the parents) and child's information (name, gender, birth date, place of delivery, ethnicity and types of household register) were collected and included in the analyses as potential confounding factors. Records on vaccination certificates kept by parents or vaccination cards kept in IC, rather than parents' memories of vaccination, were extracted as evidence of vaccine immunisation to avoid possible recall bias. Children were considered unvaccinated if both the vaccination certificate and vaccination card were absent. It took about $10 \mathrm{~min}$ to complete a questionnaire. Newborns who received a HepB in the first and second day of birth were deemed to finish the vaccine within 24 hours and have a timely HepB first dose in our analysis, ${ }^{1}$ because it is hard to calculate the exact hours that the babies receive the vaccine.

A total of 42 children were surveyed in each township, including 10 from the village where the township government was located and 8 each from the remaining four villages. Ultimately, a total of 1428 children were selected in the province. Because this was part of regular national public health evaluation of EPI coverage by the township level, all selected subjects should be surveyed, therefore the response rate in our study was $100 \% .{ }^{18}$ The sampling method was identified as a suitable method for evaluation of the EPI coverage rate by the township level and the sample (42 children) of each township had a power of $90 \%$ to detect whether the coverage rate of EPI reached $90 \%$ with a 0.05 significance level. ${ }^{18}$ The study was part of a national activity which was organised by the China NHFPC to evaluate whether the EPI coverage by the township level meets the requirements of the 12th Five Year Plan of Health Development and was exempt from ethical review. ${ }^{16}$ 


\section{Outcomes}

The primary outcome was completion rate of the EPI, which was defined as the percentage of children who had finished the EPI. Completion of the EPI was defined as children completing the EPI according to the recommended immunisation schedule. Children aged 2-3 years should finish one dose of BCG, three doses of HepB, three doses of OPV, four doses of DTaP, two doses of MCV, two doses of MPSV-A, one dose of HepA-L and the first dose of JE-L and an additional second dose of JE-L was demanded for children of 3-4 ages. Vaccination earlier than the initiation schedules time of vaccines and with incorrect intervals between different doses of vaccines was defined as ineffective vaccination.

Children who had received the SFV in accordance with immunisation schedule were identified as completing the corresponding EPI vaccines. The equivalences between SFV and EPI vaccines were as follow ${ }^{19}$ : (1) For HepB, DTaP, OPV and MPSV-A, one dose of SFV was equivalent to one dose of EPI vaccine. (2) For JE-L, two doses of inactivated Japanese encephalitis vaccine (JE-I) should be vaccinated to 3-year-old children and a third JE-I dose be given to 4-year-old children, which were equal to the first dose and second dose of JE-L, respectively. (3) For HepA-L, one dose of inactivated hepatitis A vaccine (HepA-I) was equal to one dose of HepA-L for children aged below 3 years. However, children who had received a dose of HepA-I should accept another HepA-I dose by 4 years of age, which was identified as completion of the HepA-L for children aged 3-4 years.

The secondary outcome was the coverage of SFV, which was defined as the percentage of children who had accepted one or more doses of the SFV. In this study, the SFV included IPV (substitute for OPV), DTaP and Haemophilus influenzae type $\mathrm{B}$ (HIB) conjugate vaccine (substitute for DTaP), DTaP/IPV/HIB conjugate vaccine (substitute for OPV and DTaP), group A/C meningococcal conjugate vaccine (substitute for MPSV-A), hepatitis $\mathrm{A}$ and $\mathrm{B}$ combination vaccine (substitute for HepA-L and HepB), HepA-I (substitute for HepA-L) and JE-I (substitute for JE-L).

\section{Statistical analyses}

Data were double entered using EpiData V.3.1 to ensure the consistency and avoid information bias. Multivariate logistic regression models were fitted to estimate the ORs and their 95\% CIs for completion of the EPI, after controlling for the potential confounding factors. Pearson's correlation coefficient was evaluated to intuitively reflect the correlation between the EPI completion rate and the SFV coverage rate in regions with the SFV supply.

Due to the implementation of different policies, SFV for children were banned in some areas. To compare completion rates among children in regions with SFV access to Fujian as a whole, we also ran a multivariate logistic regression analysis in the regions of Fujian with access to the SFV. Children were further divided into three groups, including children living in areas without
SFV access (these children did not receive SFV because there was no SFV in the areas), children without history of SFV vaccination in areas with SFV access and children who received the SFV in areas with SFV access. Logistic regression models were remodelled to compare the EPI coverage rates among the three groups.

To examine whether the relationship between receipt of the SFV and completion of the EPI was modified by parents' education level and initiation time of the HepB first dose, we performed cumulative completion rate stratification analysis based on parents' education level (junior school or less vs high school or above) and initiation time of the HepB first dose (> vs $\leq 24$ hours) by Cox proportional hazards analyses, in which the EPI completion time (months) was set as 'survival time'.

All statistical tests were conducted using SPSS V.19.0. A $p$ value of $<0.05$ was considered statistically significant.

\section{RESULTS}

\section{Characteristics of the study population}

In total, 1428 children of an appropriate age were surveyed, of whom 1350 (94.5\%) finished the EPI, and $282(19.7 \%)$ received at least of one dose of the SFV. Most of the children were of Han ethnicity $(97.8 \%)$ and were local children $(89.6 \%)$. More than half of the children were boys $(54.0 \%)$ and aged 2 years $(56.2 \%)$; and most children $(85 \%)$ were born in hospitals at the county level and above.

Table 1 shows the population characteristics of the entire sample and the selected children in regions with the SFV access, stratified by children with and without completion of the EPI. Children who had a history of the SFV were more likely to complete the EPI than those who had not received the SFV (97.9\% vs $93.7 \%$ ) in total sample, as well as in the selected sample (97.9\% vs $92.1 \%)$.

\section{Receipt of the SFV and completion of the EPI}

Compared with children who did not receive the SFV, children who had administrated the SFV were more likely to complete the EPI (unadjusted OR 3.1, 95\% CI 1.3 to 7.2 and adjusted OR $3.1,95 \%$ CI 1.3 to $7.4 ; \mathrm{p}=0.009$ ) in the total sample. The adjusted OR rose to 3.9 (95\% CI 1.6 to 9.4 ) in the subgroup of children in regions with the SFV supply (table 2). Children of 3 years old and with timely initiation of the HepB first dose $(<24$ hours after birth) were more likely to complete the EPI than those of 2 years old and with a delayed HepB first dose. The minority children were less likely to complete the EPI than the Han children (table 2). Educational level of the child's father also affected the completion of EPI in the total sample but insignificant in the subgroup sample (table 2). The SFV coverage was correlated with the EPI completion rate in children from regions with the SFV access (correlation coefficient $=0.689 ; \mathrm{p}=0.059$ ) (figure 1).

After controlling for potential factors (child's gender, age and nationality, parents' education level, etc), 
Table 1 Base characteristics of children in groups with and without completion of the EPI in total and selected sample

\section{Total sample \\ ( $n=1428)$}

Characteristics

History of the SFV administration

\begin{tabular}{|c|c|c|c|c|}
\hline No & $72(6.3)$ & 1074 (93.7) & $57(7.9)$ & 669 (92.1) \\
\hline Yes & $6(2.1)$ & $276(97.9)$ & $6(2.1)$ & $276(97.9)$ \\
\hline \multicolumn{5}{|l|}{ Gender } \\
\hline Male & $40(5.2)$ & 731 (94.8) & $32(5.8)$ & 517 (94.2) \\
\hline Female & $38(5.8)$ & $619(94.2)$ & $31(6.8)$ & $428(93.2)$ \\
\hline \multicolumn{5}{|l|}{ Age at survey (years) } \\
\hline $2 \sim$ & $56(7.3$ & 746 (92.7) & $45(8.2)$ & $502(91.8)$ \\
\hline $3 \sim$ & $22(3.4)$ & $634(96.6)$ & $18(3.9)$ & $443(96.1)$ \\
\hline \multicolumn{5}{|l|}{ Nationality } \\
\hline Han & $70(5.0)$ & $1327(95.0)$ & $55(5.6 \%)$ & $922(94.4)$ \\
\hline Minority & $8(25.8)$ & $23(74.2)$ & $8(25.8)$ & $23(74.2)$ \\
\hline \multicolumn{5}{|l|}{ Place of birth } \\
\hline County hospital or above & $65(5.4)$ & 1149 (94.6) & $54(6.5)$ & 777 (93.5) \\
\hline Township hospital & $12(5.7)$ & $197(94.3)$ & $8(4.6)$ & 165 (95.4) \\
\hline Home & $1(20.0 \%)$ & $4(80.0 \%)$ & $1(25.0 \%)$ & $3(75.0 \%)$ \\
\hline \multicolumn{5}{|l|}{ Household registration } \\
\hline Local registration & $69(5.4)$ & $1211(94.6)$ & $54(6.2)$ & $819(93.8)$ \\
\hline Outside or no registration & $9(6.1)$ & 139 (93.9) & $9(6.7)$ & $126(93.3)$ \\
\hline \multicolumn{5}{|l|}{ Mother's educational level } \\
\hline Junior school or less & $68(6.6)$ & 967 (93.4) & $56(7.9)$ & $652(92.1)$ \\
\hline High and technical school & $8(3.2)$ & $240(96.8)$ & $6(3.1)$ & $189(96.9)$ \\
\hline College or higher & $2(1.4)$ & $143(98.6)$ & $1(1.0)$ & 104 (99.0) \\
\hline \multicolumn{5}{|l|}{ Father's educational level } \\
\hline Junior school or less & $69(6.9)$ & $925(93.1)$ & $55(8.0)$ & $629(92.0)$ \\
\hline High and technical school & $7(2.6)$ & $261(97.4)$ & $7(3.4)$ & 197 (96.6) \\
\hline College or higher & $2(1.2)$ & $164(98.8)$ & $1(0.8)$ & $119(99.2)$ \\
\hline \multicolumn{5}{|l|}{$\begin{array}{l}\text { Initiation time of the hepatitis B } \\
\text { vaccine first dose }\end{array}$} \\
\hline$>24$ hours & $9(13.2)$ & $59(86.8)$ & $9(17.6)$ & $42(82.4)$ \\
\hline$\leq 24$ hours & $69(5.1)$ & 1291 (94.9) & 54 (5.6) & 903 (94.4) \\
\hline
\end{tabular}

EPI, expanded programme on immunisation; SFV, substitutable-for-fee vaccines. compared with children in regions without the SFV access, children who had received the SFV were more likely to complete the EPI (OR 1.8 ; $95 \%$ CI 0.7 to 4.8 , $\mathrm{p}=0.25$ ), while those without the SFV administration were less likely to complete the EPI (OR 0.5 ; $95 \%$ CI 0.2 to $0.8, \mathrm{p}=0.01$ ) in regions with the SFV supply.

\section{Stratified analyses}

We observed significant differences of EPI coverage rates between groups of children with and without the SFV administration by stratifications of parents' educational level (junior school or less vs high school or above) and initiation time of the HepB first dose ( $>$ vs $\leq 24$ hours
Population in regions with the SFV access ( $n=1008)$

Did complete EPIN (\%) (\%)

(\%)
Did not complete EPIn Did complete EPIn

$517(94.2)$

$32(5.8)$

$428(93.2)$

$502(91.8)$

$43(96.1)$

$922(94.4)$

$3(74.2)$

$777(93.5)$

$65(95.4)$

cantly higher cumulative completion rate than those without the SFV administration in the subgroup of children whose parents with education level of junior school or less $(97.8 \%$ vs $92.5 \%$ for mother's education level and $97.9 \%$ vs $91.9 \%$ for father's education level, both $\mathrm{p}<0.001$ ) (figure 2B, D). Similarly, children with timely initiation of a HepB first dose were more sensitive to the SFV vaccination, the cumulative EPI completion rate was higher among children receiving the SFV than that among those without the SFV administration $(98.5 \%$ vs $94.0 \%, \mathrm{p}<0.001$ ) (figure $2 \mathrm{E}$ ). No significant result was 
Table 2 ORs and $95 \%$ Cls for completion of EPI

\begin{tabular}{|c|c|c|c|c|}
\hline \multirow[b]{2}{*}{ Characteristics } & \multicolumn{2}{|c|}{ Total sample $(n=1428)$} & \multicolumn{2}{|c|}{$\begin{array}{l}\text { Population in regions with the SFV } \\
\text { access }(n=1008)\end{array}$} \\
\hline & ORs (95\% Cl) & p Value & ORs $(95 \% \mathrm{Cl})$ & $\mathrm{p}$ Value \\
\hline \multicolumn{5}{|c|}{ History of the SFV administration } \\
\hline No & Reference & & Reference & \\
\hline Yes & 3.1 (1.3 to 7.4$)$ & 0.009 & 3.9 (1.6 to 9.4$)$ & 0.002 \\
\hline \multicolumn{5}{|l|}{ Gender } \\
\hline Male & Reference & & Reference & \\
\hline Female & 0.8 (0.5 to 1.4$)$ & 0.48 & $0.8(0.5$ to 1.4$)$ & 0.46 \\
\hline \multicolumn{5}{|l|}{ Age at survey (years) } \\
\hline $2 \sim$ & Reference & & Reference & \\
\hline $3 \sim$ & 2.5 (1.5 to 4.2$)$ & 0.001 & 2.5 (1.4 to 4.5$)$ & 0.002 \\
\hline \multicolumn{5}{|l|}{ Nationality } \\
\hline Han & Reference & & Reference & \\
\hline She & $0.2(0.1$ to 0.4$)$ & $<0.001$ & $0.2(0.1$ to 0.5$)$ & 0.001 \\
\hline \multicolumn{5}{|l|}{ Place of birth } \\
\hline County hospital or above & Reference & & Reference & \\
\hline Township hospital & 1.1 (0.6 to 2.2$)$ & 0.74 & 1.9 (0.9 to 4.2$)$ & 0.10 \\
\hline Home & 0.5 (0.05 to 5.8$)$ & 0.60 & 0.7 (0.06 to 9.2$)$ & 0.79 \\
\hline \multicolumn{5}{|l|}{ Household registration } \\
\hline Local registration & Reference & & Reference & \\
\hline Outside or no registration & 0.8 (0.4 to 1.6$)$ & 0.46 & 0.9 (0.4 to 1.9$)$ & 0.71 \\
\hline \multicolumn{5}{|l|}{ Mother's educational level } \\
\hline Junior school or less & Reference & & Reference & \\
\hline High and technical school & 1.1 (0.5 to 2.5$)$ & 0.83 & 1.4 (0.5 to 3.8$)$ & 0.50 \\
\hline College or higher & 1.7 (0.3 to 9.4$)$ & 0.55 & 2.7 (0.3 to 25.8$)$ & 0.38 \\
\hline \multicolumn{5}{|l|}{ Father's educational level } \\
\hline Junior school or less & Reference & & Reference & \\
\hline High and technical school & 2.4 (1.0 to 5.7$)$ & 0.054 & 1.9 (0.7 to 4.7$)$ & 0.18 \\
\hline College or higher & 4.5 (0.8 to 24.8$)$ & 0.09 & 6.5 (0.7 to 62.4$)$ & 0.10 \\
\hline \multicolumn{5}{|l|}{$\begin{array}{l}\text { Initiation time of the hepatitis B } \\
\text { vaccine first dose }\end{array}$} \\
\hline$>24$ hours & Reference & & Reference & \\
\hline$\leq 24$ hours & $2.7(1.2$ to 6.0$)$ & 0.016 & $3.9(1.7$ to 9.3$)$ & 0.002 \\
\hline
\end{tabular}

EPI, expanded programme on immunisation; SFV, substitutable-for-fee vaccines.

observed for children whose parents have education of high school and above and who had received a delayed HepB first dose (figure 2A, C, F).

\section{DISCUSSION}

We found that children who had received SFV were more likely to complete the EPI, where the association may be offset by high parents' education level and the delayed initiation of the HepB first dose. In China, frequent reports of AEFI, such as the Shanxi vaccine incident $^{5}$ and the Conde HepB event, ${ }^{6}$ had further solidified the parents' views of 'cheap is dear, and dear is cheap', they would choose the SFV even if they have to pay an extra cost (each dose costs $¥ 18$ to $¥ 630$ mean: $¥ 156 /$ dose, approximately US $\$ 24.6 /$ dose $^{20}$ ). Moreover, receiving the SFV may create revenue and reduce the incidence of AEFI caused by the EPI vaccines, ${ }^{11} 21$ therefore, children who chose the SFV might be treated more enthusiastically by the staff in the IC than those who chose the EPI vaccines. Hence, receiving the SFV may be an indicator of the economic conditions and vaccination awareness of children' families, and might 


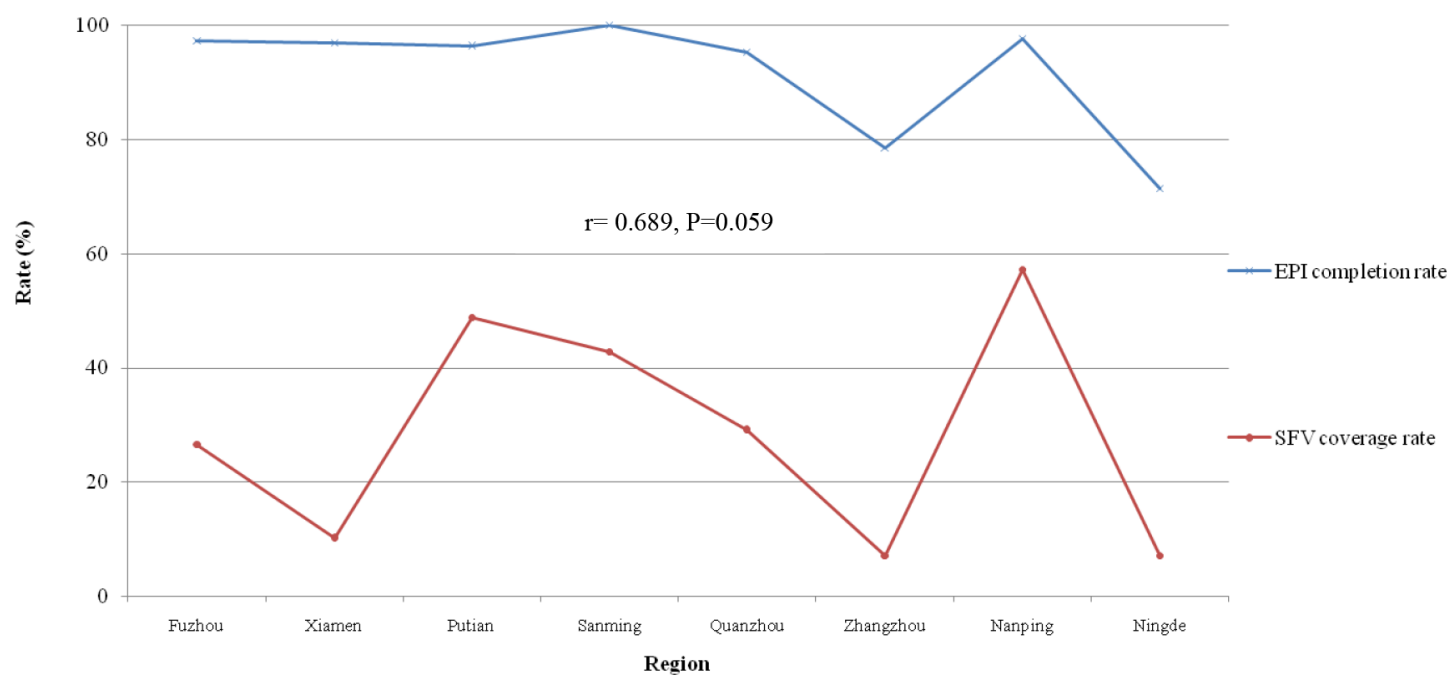

Figure 1 The correlation analysis between the EPI completion rate and the SFV coverage rate in regions with the SFV supply. EPI, expanded programme on immunisation; SFV, substitutable-for-rfee vaccines.

ensure better service from the staff of the IC, resulting in children having better compliance with immunisation and being more likely to complete the EPI. ${ }^{12922-26}$

Parents with lower education level have certain characteristics (earn less, lack of knowledge and have multiple children) that are not conducive to complete the EPI for their children ${ }^{27}$; ; they might be more sensitive to the price of the $\mathrm{SFV}^{27-29}$ Whether to receive the $\mathrm{SFV}$ may further reflect their economic situation and immunisation awareness, which leads to the difference of the EPI completion between the two groups. The HepB first dose are often delayed as a result of contraindications for children born in hospitals or of low medical accessibility for those born at home, ${ }^{130} 31$ which might affect their catch-up vaccination of the EPI vaccines even if they had received the SFV, resulting in a significantly lower completion of the EPI than those with timely initiation of the HepB first dose.

Interestingly, we found that the supply of the SFV may play a 'double-sword' role in the EPI completion. Vaccine providers may differentiate children who had received the EPI vaccines from those with the SFV, which may lead to a decline in the enthusiasm of the completion of the EPI. ${ }^{32}$ Moreover, the difference of completion rate of the EPI between regions with and without the SFV supply may be partly explained by the percentage of non-local children, which was proved as an impact factor for the EPI completion. ${ }^{24}{ }^{31}$ In our study, the percentage of non-local children was significantly lower in regions without SFV supply than that in regions with the SFV access $(3.1 \%$ vs $13.4 \%, \mathrm{p}<0.001)$.

We also found that the EPI completion rate was higher in children aged 3 years, which differed from findings of a previous study. ${ }^{31}$ This inconsistency may be explained due to the fact that the age of 3 is usually the start to attend kindergartens, which are required to examine the vaccination certificate of children under regulations, therefore prompting children to receive their missing vaccinations $^{33}$ and effectively improving the completion of the EPI in 3-year-old children. Minority children were less likely to complete the EPI, which may be related to poor economic conditions of their families ${ }^{34}$ and the lack of vaccination awareness of their parents. ${ }^{35}$ Similar to previous studies, ${ }^{36}{ }^{37}$ we confirmed that timely HepB first dose initiation improved the EPI completion in children, possibly because receiving a timely HepB first dose might help emphasise the importance of vaccination to the parents and promote their children' completion of the EPI. ${ }^{1}$

Our study has several limitations. First, we included the $\mathrm{SFV}$, but not normal for-free vaccines, such as the varicella vaccine and the influenza vaccine, which might introduce certain information bias. However, most normal for-fee vaccines are not administered until children are 2 years old, this bias might not affect the results of this study. Second, the moderate sample size of this study may limit the interpretation of some results. Finally, other factors, such as access to healthcare, knowledge, attitudes and the practices of parents and providers, regional vaccination policy, the level of regional economic development, will also affect the SFV vaccination. In addition, sociodemographic variables, such as the number of children, parent age, family income and so on, might be better indicators of the willingness to receive SFV than the parents' education level. Unfortunately, we were not able to include all these factors in the current study.

To the best of our knowledge, this is the first study exploring the association between the SFV administration and the EPI completion in Fujian, China. The association between the SFV administration and the EPI completion was more obvious among children whose parents have lower education level and among those with a timely HepB first dose. Vaccine providers should focus on these vulnerable children and take measures to help 
A

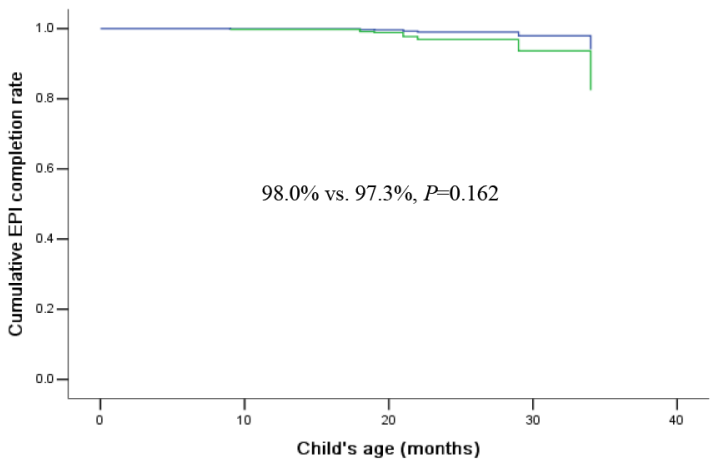

$\mathrm{C}$

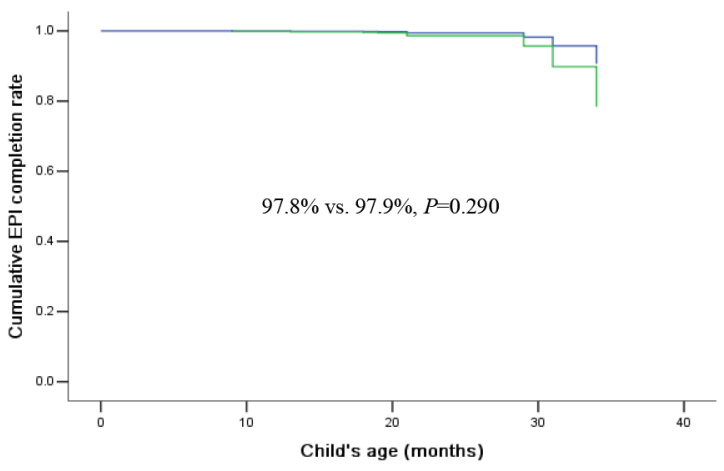

E Children with a timely first dose of the hepatitis B vaccine

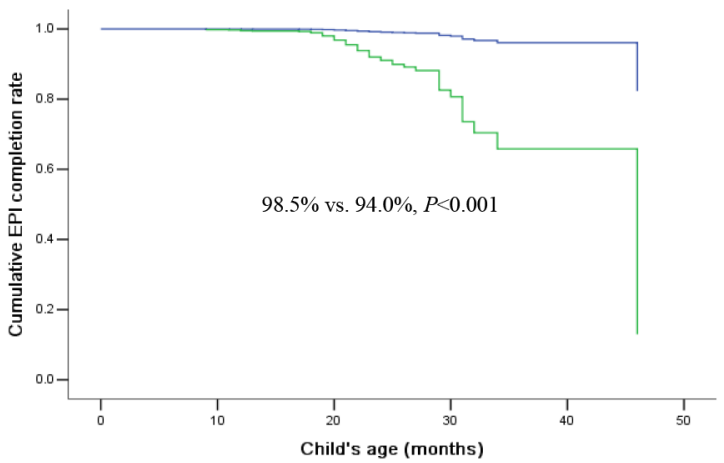

B

Children whose mother has education of junior school or less

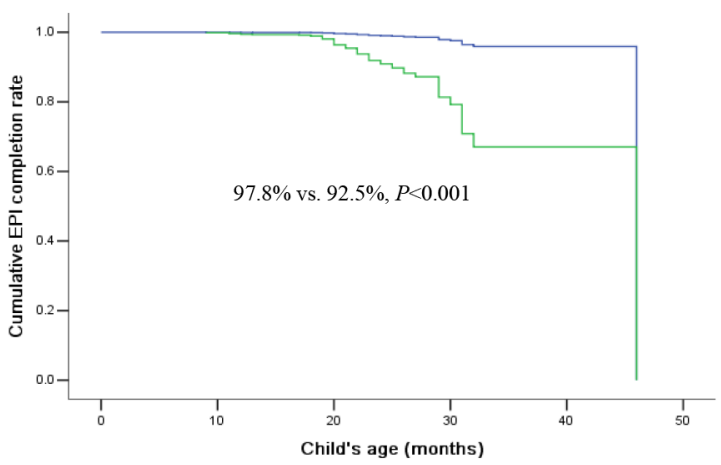

D Children whose father has education of junior school or less

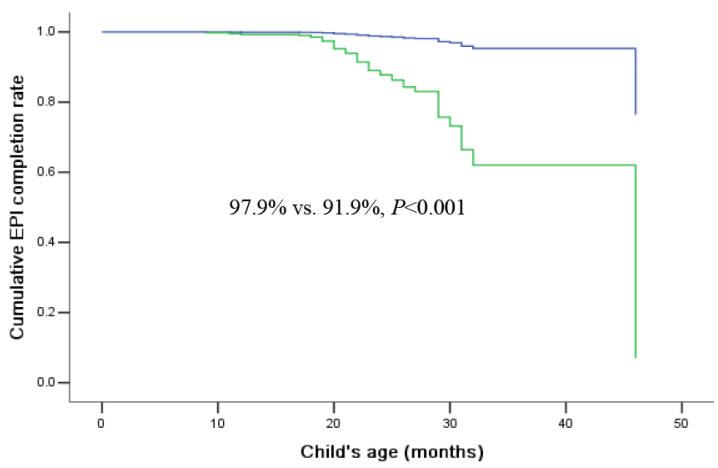

F Children with a delayed first dose of the hepatitis B vaccine

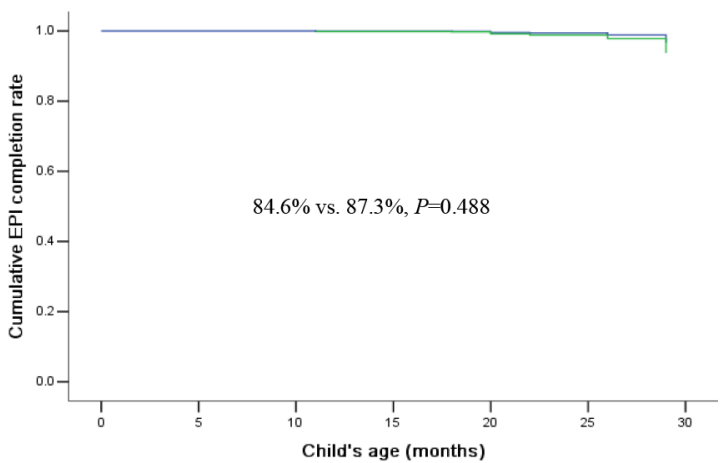

Figure 2 Cox proportional hazards models estimates of the EPI cumulative completion rate according to the receipt of the SFV, stratified by parents' education level and initiation time of the hepatitis B vaccine first dose. A, B, C and D show the difference of cumulative EPI completion rate between groups of children with and without the SFV vaccination by parents' education level (high school and above vs junior school or less), as well as by initiation time of the hepatitis B vaccine first dose (timely vs delayed initiation) in E and F. EPI, expanded programme on immunisation; SFV, substitutable-for-fee vaccines.

them complete the EPI on time. The generalisability of the results should be further studied.

Acknowledgements The authors appreciate the assistance and cooperation of the local field investigators and the survey participants. The authors also thank Andrew Smith (Excellence in Learning For All [ELFA]A Juyuan preschool of Pudong) for his review of the manuscript.
Contributors JNW, YZ and DJL had the initial idea for the study and designed the survey. JNW, MRD and HLP collected and analysed the data for this study. JNW wrote the first draft of the manuscript. All authors critically revised the manuscript and approved the final version.

Competing interests None declared.

Patient consent Obtained.

Ethics approval Chinese Center for Disease Control and Prevention. 
Provenance and peer review Not commissioned; externally peer reviewed.

Data sharing statement No additional data are available.

Open Access This is an Open Access article distributed in accordance with the Creative Commons Attribution Non Commercial (CC BY-NC 4.0) license, which permits others to distribute, remix, adapt, build upon this work non-commercially, and license their derivative works on different terms, provided the original work is properly cited and the use is non-commercial. See: http://creativecommons.org/ licenses/by-nc/4.0/

(c) Article author(s) (or their employer(s) unless otherwise stated in the text of the article) 2017. All rights reserved. No commercial use is permitted unless otherwise expressly granted.

\section{REFERENCES}

1. Wu JN, Li DJ, Zhou Y. Association between timely initiation of hepatitis $B$ vaccine and completion of the hepatitis $B$ vaccine and national immunization program vaccine series. Int $\mathrm{J}$ Infect Dis 2016;51:62-5.

2. Zheng JS, Cao L, Guo SC, et al. Survey on the immunization status of category $B$ vaccine among children aged 1 to 2 years in China. Chin J Vacci Immuni 2012;18:233-7.

3. The State Council, P.R. China. Regulation on vaccine circulation and immunization management (Revised). 2016. http://news.xinhuanet. com/politics/2016-04/25/c 1118730528.htm

4. National Health and Family Planning Commission of the People' Republic of China. Notification of regulating the work of the expanded national immunization program. http://www.nhfpc.gov.cn/j kj/s3581/201505/b12b420d05f34a748c55d84d93f06d21.shtml

5. Zhang SY. Enlightenment from the vaccine of Shanxi province. Chin $J$ PHM 2010;26:578-9.

6. Yue CY, Sun XJ, Wei N, et al. Quick assessment on the influence of hepatitis B vaccine event reported by media to children's parents in 2013. Chin J Vacci Immuni 2014;20:100-4.

7. Lin ZQ, Rh W, Jn W, et al. Study on the incidence of vaccineassociated paralytic poliomyelitis and its influencing factors in Fujian province, 2008-2011. Chin J Epidemiol 2013;34:413-4.

8. Demand GB. need, and affecting factors on immunization service. Chin J Vacci Immuni 2007;13:182-5.

9. Hu Y, Luo S, Tang X, et al. Comparative assessment of immunization coverage of migrant children between national immunization program vaccines and non-national immunization program vaccines in East China. Hum Vaccin Immunother 2015;11:761-8.

10. Hu Y, Chen Y, Zhang B. Two-dose seasonal influenza vaccine coverage and timeliness among children aged 6 months through 3 years: an evidence from the 2010-11 to the 2014-15 seasons in Zhejiang province, east China. Hum Vaccin Immunother 2017;13:75-80.

11. Hou Z, ChangJ, Yue D, et al. Determinants of willingness to pay for self-paid vaccines in China. Vaccine 2014;32:4471-7.

12. Yang HY. Study on the coverage of self-paid vaccines among children aged 2 to 6 years in Maoming City. South China J Prev Med 2015;41:58-60.

13. Hontelez JA, Hahné SJ, Oomen P, et al. Parental attitude towards childhood HBV vaccination in The Netherlands. Vaccine 2010;28:1015-20.

14. Smith PJ, Jain N, Stevenson J, et al. Progress in timely vaccination coverage among children living in low-income households. Arch Pediatr Adolesc Med 2009;163:462-8.

15. Wang YY, Wang Y, Zhang JX, et al. Status of mother's KAP on child immunization on minority areas, Guizhou Province. Beijing Da Xue Xue Bao 2007;39:136-9.

16. Zheng JS, Cao L, Cao LS, et al. Immunization coverage of the national immunization program vaccines at the township level, based on a survey conducted by provincial CDCs in China, 2013. Chin J Vacci Immuni 2014;6:492-546.

17. Cao L, Zheng JS, Cao LS, et al. Immunization coverage of national immunization program vaccines at the township level, based on a survey by the county level, China, 2013. Chin J Vacci Immuni 2014;6:486-91.

18. Cao L, Zheng JS, Sc Y, et al. Sampling design of the China immunization coverage survey for national immunization program vaccines at the township level in 2013. Chin J Vacci Immuni 2014;20:481-5

19. National Health and Family Planning Commission of the People' Republic of China. Notification of the immunization schedules and instructions of the national immunization program for children. 2016. http://www.nhfpc.gov.cn/jkj/s3581/201701/a91fa2f3f9264cc186e1d ee4b1f24084.shtml

20 . Xiamen Centers for Disease Control and Prevention. Purchase and sale price of self-paid vaccines in 2014-2015 in Xiamen City (EB/ OL).

21. Zhang $L, A q X$, Song $L Z$, et al. Investigation on knowledge, attitude and practice (KAP) about adverse events following immunization (AEFI) among staff on immunization program in Shandong Province, China. Chin J Vacci Immuni 2006;12:300-2.

22. Ganczak M, Dmytrzyk-Daniłów G, Karakiewicz B, et al. Determinants influencing self-paid vaccination coverage, in $0-5$ years old Polish children. Vaccine 2013;31:5687-92.

23. Dorell CG, Yankey D, Santibanez TA, et al. Human papillomavirus vaccination series initiation and completion, 2008-2009. Pediatrics 2011;128:830-9.

24. Johri M, Pérez MC, Arsenault C, et al. Strategies to increase the demand for childhood vaccination in low- and middle-income countries: a systematic review and meta-analysis. Bull World Health Organ 2015;93:339-46.

25. Canavan ME, Sipsma HL, Kassie GM, et al. Correlates of complete childhood vaccination in east african countries. PLoS One 2014;9:e95709.

26. Awoh $A B$, Plugge $E$. Immunization coverage in rural-urban migrant children in low and middle-income countries (LMICs): a systematic review and meta-analysis. J Epidemiol Community Health 2016;70.

27. Branco FL, Pereira TM, Delfino BM, et al. Socioeconomic inequalities are still a barrier to full child vaccine coverage in the Brazilian Amazon: a cross-sectional study in Assis Brasil, Acre, Brazil. Int J Equity Health 2014;13:118-29.

28. Racine AD, Joyce TJ, education M. Maternal education, child immunizations, and public policy: evidence from the US National Immunization Survey. Soc Sci Med 2007;65:1765-72.

29. Nankabirwa V, Tylleskär T, Tumwine JK, et al. Maternal education is associated with vaccination status of infants less than 6 months in Eastern Uganda: a cohort study. BMC Pediatr 2010;10:92.

30. Cui FQ, Gong XH, Chen YS, et al. Evaluation on impact of hepatitis $B$ vaccine integrated into routine immunization in the areas of Ministry of Health/Global Alliance for Vaccine and Immunization (GAVI) Cooperation Project PR China. Zhongguo Yi Miao He Mian Yi 2009;15:289-93.

31. Liang X, Bi S, Yang W, et al. Evaluation of the impact of hepatitis B vaccination among children born during 1992-2005 in China. J Infect Dis 2009;200:39-47.

32. Yu YX, Huang MD, JH L. Status and influencing factors on vaccination of preschool children in Foshan. Chin Primary Health Care 2015;29:59-61.

33. Jn W, Huang LF, Lin ZQ. Study on coverage rates of the EPI vaccines and its influencing factors in Fujian, China. Strait J Prev Med 2015;21:1-3.

34. Whitehead SJ, Cui KX, De AK, et al. Identifying risk factors for underimmunization by using geocoding matched to census tracts: a statewide assessment of children in Hawaii. Pediatrics 2007;120:e53 5-e542.

35. Wang YY, Wang Y, Zhang JX, et al. Status of mother's KAP on child immunization on minority areas, Guizhou Province. Beijing Da Xue Xue Bao 2007;39:136-9.

36. Wu JN, Wen XZ, Zhou Y, et al. Impact of the free-vaccine policy on timely initiation and completion of hepatitis $B$ vaccination in Fujian, China. J Viral Hepat 2015;22:551-60.

37. Yusuf HR, Daniels D, Smith P, et al. Association between administration of hepatitis $B$ vaccine at birth and completion of the hepatitis $B$ and 4:3:1:3 vaccine series. JAMA 2000;284:978-83. 